J. A. ROSANOW

STOCHASTISCHE PROZESSE 
MATHEMATISCHE LEHRBÜCHER UND MONOGRAPHIEN

HERAUSGEGEBEN VON DER

AKADEMIE DER WISSENSCHAFTEN DER DDR

ZENTRALINSTITUT FÜR MATHEMATIK UND MECHANIK

I. ABTEILUNG

MATHEMATISCHE LEHRBƯCHER

BAND 28

STOCHASTISCHE PROZESSE

VON

J. A. ROSANOW

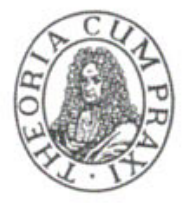

AKADEMIE-VERLAG · BERLIN

1975 


\author{
J. A. ROSANOW
}

\title{
STOCHASTISCHE PROZESSE
}

\author{
EINE EINFÜHRUNG
}

In deutscher Sprache herausgegeben

von

Dr. UWE KÜCHLER

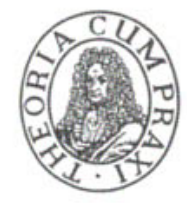

AKADEMIE-VERLAG - BERLIN

1975 
Ю. А. Розанов

Случайные процессы

Erschienen im Verlag Nauka, Moskau

Deutsche Übersetzung: Dipl.-Math. Jürgen Franz, Dr. Lothar Partzsch

Erschienen im Akademie-Verlag, 108 Berlin, Leipziger Straße 3-4

(c) Akademie-Verlag, Berlin, 1975

Lizenznummer: $202 \cdot 100 / 407 / 75$

Gesamtherstellung: VEB Druckhaus „Maxim Gorki“", 74 Altenburg

Bestellnummer: 7621234 (6160) · LSV 1074

Printed in GDR

EVP 54,- 